Recepción: 15 de febrero de 2017

Aceptación: 15 de marzo de 2017

Publicación: 14 de junio de 2017

\title{
ESTUDIO ORGANIZACIONAL DEL CLOUD COMPUTING EN EMPRESAS EMPRENDEDORAS
}

\section{CLOUD COMPUTING ORGANIZATIONAL STUDY IN ENTREPRENEURSHIPS}

Pedro R. Palos-Sánchez ${ }^{1}$

1. Doctor en Economía y Profesor del Departamento de Dirección de Empresas y Sociología. Universidad de Extremadura, Cáceres. España. E-Mail: ppalos@unex.es

\section{Citación sugerida:}

Palos-Sánchez, P.R. (2017). Estudio organizacional del Cloud Computing en empresas emprendedoras. 3C Tecnología: glosas de innovación aplicadas a la pyme, 6(2). 1-16. DOI: $<$ http://dx.doi.org/10.17993/3ctecno.2017.v6n2e22.1-16/>. 


\section{RESUMEN}

El Cloud Computing es considerado como un nuevo modelo de negocio en sí, y está permitiendo que muchas empresas trasladen su actividad offline a online y que muchos emprendedores encuentren en esta tecnología el mejor soporte de su negocio, sin tener que llevar a cabo inversiones costosas, debido a las características de la tecnología y a su modelo de despliegue. Sin embargo, las incertidumbres y barreras con las que se enfrentan hacen que las empresas desconfíen por razones de privacidad, seguridad y, en algunos casos, razones de índole legal. Este trabajo está escrito con el objetivo de conocer el uso que hacen las organizaciones de esta tecnología, analizando, especialmente, el grado de confianza que depositan en la tecnología y las ventajas organizacionales que aporta.

A través de una encuesta a empresas emprendedoras, se encontró que la mayoría de las pymes usan la nube para tareas de ofimática y almacenamiento de ficheros y bases de datos, lo que significa que los servicios Cloud que usan requieren de confianza y seguridad. Entre las ventajas encontradas, para la organización, están la mejora de la productividad en los procesos, una mayor flexibilidad derivada de la utilización de los recursos tecnológicos y el ahorro de costes que supone aumentar la capacidad informática sin que lo haga el resto de la organización.

\section{ABSTRACT}

Cloud computing is considered as a new business model in itself, and it is allowing many companies to move their activity offline to online and that many entrepreneurs find in this technology the best support of their business, without having to carry out expensive investments due To the characteristics of the technology and to its model of deployment. However, the uncertainties and barriers they face make companies suspicious for reasons of privacy, security and, in some cases, legal reasons. This work is written with the objective of knowing the use that the organizations make of this technology, analyzing, especially, the degree of confidence that they deposit in the technology and the organizational advantages that it contributes.

Through a survey of entrepreneurial companies, it was found that most SMBs use the cloud for office tasks and storage of files and databases, which means that the Cloud services they use require trust and security. Among the advantages found for the organization are the improvement of productivity in the processes, greater flexibility derived from the use of technological resources and the cost savings of increasing the computer capacity without the rest of the organization. 


\section{PALABRAS CLAVE}

Cloud Computing, Software como Servicio (Saas), Emprendimiento, Modelo de negocio, Seguridad, Privacidad, Confianza, Protección de datos.

\section{KEY WORDS}

Cloud Computing, Software as a Service (SaaS), Entrepreneurship, Business model, Security, Privacy, Trust, Data Protection. 


\section{INTRODUCCIÓN}

El Cloud Computing (computación en la nube) o simplemente la nube, está evolucionando de manera significativa en los últimos años y está siendo implantado por las empresas. Se trata de una alternativa al modelo de implementación tradicional de las TIC (Tecnologías de la Información y las Comunicaciones), donde se ha pasado de comprar los recursos informáticos a pagar por su uso (Marston et al., 2011). Por tanto, se está demostrando en todo el mundo una gran transformación tecnológica, tanto en el ámbito de los negocios como en el de los usuarios individuales (Arinze y Anandarajan, 2010).

El presente trabajo tiene como objetivo conocer el uso que hacen las empresas de Cloud Computing. Se pretende estudiar cuál es el grado de uso de esta tecnología en las empresas y sus ventajas e incertidumbres aplicadas a los procesos. Por último, se analizó la confianza que depositan las empresas en esta tecnología basada en la nube.

En cuanto a la estructura del documento, en primer lugar se definirá el concepto y características del cloud computing así como diversos aspectos teóricos sobre esta tecnología. En una segunda parte, se expone la metodología, que consiste en la realización de una encuesta a pymes emprendedoras. Finalmente, se exponen los resultados y se contrastan los objetivos establecidos con los resultados obtenidos, aportando una visión de la realidad del uso de Cloud Computing en las empresas emprendedoras.

\section{MARCO TEÓRICO}

\subsection{CONCEPTO}

Podemos definir el Cloud Computing como una concepción tecnológica y un nuevo modelo de negocio donde se prestan servicios de almacenamiento, acceso y uso de recursos informáticos radicados en la red.

Haciendo referencia a la definición dada por el National Institute of Standards and Technology (NIST, 2011) la computación en la nube es un modelo que permite acceso ubicuo conveniente adaptado y bajo demanda en red a un conjunto compartido de recursos configurables (redes, almacenamiento, servidores, aplicaciones y servicios) que pueden ser rápidamente aprovisionados y liberados con el mínimo esfuerzo de administración o la rápida interacción de proveedores de servicios (Mell y Grance 2011).

El concepto de Cloud Computing es cada vez más conocido y utilizado por las empresas. Genera beneficios para estas tales como la rapidez en las operaciones o cambios, el menor coste, ya que supone un ahorro en infraestructura permitiendo crear productos más potentes y mejorados lo que contribuye a una mayor estabilidad y fiabilidad. 
En este sentido, el desarrollo de tecnologías basadas en Internet es una de las estrategias cada vez más utilizada por las empresas (Tuncay, 2010). Esta tecnología ha sido estudiada como un nuevo modelo de negocio (Ross y Blumenstein, 2013; Leimeister et al., 2010, Marston et al., 2011, Yigitbasioglu et al., 2013) que permite mayor capacidad de almacenamiento, facilidad de acceso y uso de recursos o ahorro de costes. Además, los usuarios de la nube se benefician de un más adecuado uso de los recursos, en cuanto a eficiencia, flexibilidad y transferencia de riesgos (Armbrust et al., 2010).

Este nuevo modelo de negocio, suplanta a los modelos tradicionales de inversión de capital (Ross y Blumenstein, 2012). Hoy en día, la tecnología cloud facilita el desarrollo de proyectos empresariales emprendedores y de pequeñas y medianas empresas (PYMES), como por ejemplo, cuando requieren una orientación internacional. La nube proporciona un mayor acceso a los mercados globales, reduce los costos de oportunidad y apoya la colaboración y la innovación en un mundo cada vez más conectado (Ross y Blumenstein, 2015).

Pero a pesar de todo esto, existen aspectos que revelan ciertos riesgos, como la confidencialidad de los datos y la existencia de jurisdicciones extranjeras en su desarrollo y despliegue (Yigitbasioglu, 2015). Algunos estudios se han centrado en las implicaciones jurídicas (Hooper et al., 2013; Ryan y Loeffler, 2010) y otros en la privacidad y seguridad de la nube (Benlian y Hess 2010, 2011). Y por otra parte, la nube requiere superar un periodo de adopción, que no todas las organizaciones logran alcanzar (Ross y Blumenstein, 2015).

\subsection{CARACTERÍSTICAS DEL CLOUD COMPUTING}

Hay tres tipos de servicios que forman el modelo de negocio del Cloud Computing (Martín, 2014):

- Infraestructura como servicio (laaS): Referente a la oferta de almacenamiento y procesamiento en sus servidores. Únicamente está limitado por la capacidad económica de contratación del servicio que tenga el cliente.

- Plataforma como servicio (PaaS): Este servicio pone al alcance de los usuarios las herramientas para la realización de desarrollos informáticos, sin tener que adquirir e implantar en sus ordenadores locales dichas herramientas. Además, el proveedor de servicios se encarga del mantenimiento de estas herramientas informáticas.

- Software como servicio (SaaS): Se refiere a las aplicaciones finales, es decir, productos terminados que ofrecen servicios concretos. Este servicio consiste en la utilización de los servicios ofrecidos por dichas aplicaciones por parte del usuario.

En cuanto a los modelos de implementación de Cloud Computing en función de la privacidad, según la definición aportada por el NIST (2011): 
1. Nube privada: La infraestructura de este tipo de nube está preparada para el uso exclusivo de una sola organización, comprendida por varios consumidores o unidades de negocio. Puede ser de propiedad, administrado y ejecutado por la organización, por un tercero, o una combinación de ellos, y que puede existir dentro o fuera de las instalaciones.

2. Nube comunitaria: La infraestructura está preparada para el uso exclusivo de una comunidad específica de los consumidores de las organizaciones que han compartido preocupaciones, por ejemplo, la misión, requisitos de seguridad y cumplimiento... Puede ser de propiedad, administrado y ejecutado por una o más de las organizaciones que componen la comunidad, un tercero o una combinación de ellos y puede existir dentro o fuera de las instalaciones.

3. Nube pública: La infraestructura está preparada para el uso del público en general. Puede ser de propiedad, administrado y ejecutado por una empresa, academia, por instituciones del gobierno, o alguna combinación de ellos. Únicamente existe en la ubicación del proveedor del servicio.

4. Nube híbrida: La infraestructura de la nube es una combinación de dos o más tipos de las nubes anteriores, que siguen siendo entidades únicas pero están unidas por la tecnología estandarizada que permite la portabilidad de datos y aplicaciones.

\subsection{VENTAJAS Y DESVENTAJAS DEL CLOUD COMPUTING}

Entre las ventajas que tiene el cloud se pueden señalar como las más importantes (Reyna, 2009): el uso de la tecnología cloud se puede llevar a cabo desde cualquier lugar, permite el acceso a los servicios y la información, con una disponibilidad completa del servicio, es decir 24 horas y 365 días del año; además se puede acceder desde cualquier dispositivo, ya sea móvil, tablets, ordenadores portátiles u otros; los servicios son gratuitos, o pueden ser de pago dependiendo de la necesidad del usuario; al tratarse de servicios en la nube, en la web, no es necesario el almacenamiento en nuestro propio equipo por lo que permite que no se sature el disco duro.

En el apartado de las desventajas cabe señalar que la información puede estar a disposición de terceras personas, fundamentalmente técnicos; los servicios cloud dependen siempre de la existencia de conexión a Internet, lo que guarda relación con la velocidad y las tecnologías de acceso; en ocasiones el servicio es suspendido por alguna razón, ya sea un error humano o una catástrofe natural, entonces las malas repercusiones recaen sobre el cliente; almacenar datos que puedan afectar a la privacidad o fotos, videos etc. ya que se cuestiona la posible pérdida de la libertad y la privacidad del cliente, ya que estos datos pueden estar al alcance de terceras personas y todo esto puede haber problemas de descontrol del manejo de esta información (Reyna, 2009). 


\subsection{RIESGOS PRINCIPALES DEL CLOUD COMPUTING}

Gartner identifica 7 riesgos principales en el área del Cloud Computing que son la confianza en el proveedor (Brodkin, 2008), el cual debe asegurar la confidencialidad; la conformidad legal, ya que debe quedar claro que la responsabilidad de los datos o posibles infracciones legales son del proveedor que debe garantizar el cumplimiento de la normativa con el fin de dar seguridad a sus clientes; la localización de los datos o asegurar que los clientes tengan acceso a los datos; confidencialidad de la información; recuperación en caso de desastre o pérdida de datos; colaboración con la justicia aunque los datos estén en países distintos; continuidad o que se mantenga en el tiempo los datos almacenados.

\subsection{SITUACIÓN DEL CLOUD COMPUTING EN LAS EMPRESAS ESPAÑOLAS}

Maqueira-Marín y Bruque-Cámara (2012) sostienen que la cooperación entre algunos agentes como son; la Empresa, la Administración pública, y las Universidades o Instituciones de investigación, dan lugar a que sea posible el crecimiento empresarial por medio de la aplicación de la innovación y los conocimientos. Esta tendencia en la utilización de las TIC ha evolucionado hasta el término Cloud Computing, que está siendo rápidamente adoptado por el mundo empresarial.

Tomando en cuenta un estudio llevado a cabo por Urueña et al. (2012), según las previsiones analizadas en 2011, la apuesta de los proveedores de servicios tecnológicos nacionales por adoptar este modelo de servicio estaba en un punto de inflexión para la definitiva adopción del Cloud en España, debido al aumento del conocimiento que las empresas españolas van adquiriendo de los beneficios, capacidades operativas y económicas de este nuevo servicio.

Estos datos apuntan a que existe un mercado potencial para los proveedores Cloud, ya que los usuarios de internet y de telefonía móvil se sitúan como posibles consumidores potenciales de la nube (Fundación Innovación Bankinter, 2010).

A nivel Europeo y mundial, España podría ser considerada como un país idóneo para el establecimiento de centros de desarrollo y de servicios tecnológicos, ya que la combinación entre costes laborales y nivel de cualificación profesional otorgan a España, un elevado potencial para la inversión en empresas del sector TIC que estén dispuestas a implantar servicios en la nube.

Son las pequeñas y medianas empresas españolas los principales nichos y beneficiarios de los servicios Cloud, ya que ahorran en costes, posibilitan contar con infraestructura y servicios tecnológicos, así como la rapidez y agilidad en su desarrollo contribuye a que las pymes españolas puedan acceder a servicios de ERP (Enterprise Resource Planning) o CRM (Customer Relationship Management), comercio electrónico o al aumento de la capacidad de infraestructura tecnológica para el ejercicio de sus operaciones. 
Por el contrario, las grandes empresas y corporaciones de España son más reacias a la adopción del Cloud Computing, aunque están desarrollando procesos de cambio a entornos virtualizados con redes de servicios tecnológicos. Esto constituye una red de Cloud privado por lo que se prevé que en un futuro pueda ser aplicado a clientes y a otras compañías.

También las Administraciones públicas son grandes consumidoras de los servicios Cloud, debido por una parte a la optimización de la eficiencia y costes de sus servicios e infraestructuras a través de nubes privadas y públicas.

Algunos aspectos políticos públicos pueden ser también ejecutados por el sector de la información tecnológica mediante la nube, como pueden ser la modernización de la sanidad, la educación, la internacionalización de las pymes etc. Son líneas de actuación en los que la nube puede llegar a ser un instrumento crucial de dinamización y generador de eficiencia.

Hoy en día siguen existiendo algunos retos que afectan al desarrollo de este sector en España. Algunas cuestiones críticas fueron establecidas por Urueña et al. (2012) que concluyó que en el mercado español se da un gran desconocimiento de Cloud, ya que el $49 \%$ de las empresas no conoce este servicio. Así mismo, un $15 \%$ de las empresas utilizan Cloud Computing, un $9 \%$ tienen intenciones de usarlo y un $27 \%$ familiarizado con el Cloud Computing, aun no se plantea utilizarlo.

En cuanto al ritmo de adopción de este modelo, en primer lugar se sitúa el sector público y al mismo nivel, sectores como servicios, comunicación y transporte. El segundo bloque, lo constituyen, a un menor ritmo, el sector financiero y de distribución, en especial el sector financiero. Por último, encontramos al sector industrial, que es más remiso a la adopción del Cloud.

En cuanto a la adopción, las aplicaciones colaborativas y de negocio están siendo las primeras en lanzarse a los servicios Cloud, mientras que el almacenamiento y la infraestructura web están próximas a una adopción masiva (IDC, 2011). Se deduce que el crecimiento del mercado Cloud no es debido únicamente al número de empresas que empiezan a implementarlo sino también porque aquellas que lo implantan aumentan las áreas de uso.

\section{METODOLOGÍA}

La mayoría de los estudios sobre adopción de tecnología en la nube han aplicado metodologías cuantitativas, en concreto el método de la encuesta. Estos estudios se han centrado en las PYME (Alshamaila et al., 2013; Gupta et al., 2013) o industrias particulares, tales como las de alta tecnología (Low et al., 2011), sector minorista (Wu et al., 2013), turístico (Palos y Aguayo, 2016) o los sectores de la salud (Lian et al., 2014). 
Basándose en ello, el universo poblacional objeto de estudio han sido las Pymes emprendedoras, del sector servicios, de la ciudad de Sevilla, que usen normalmente tecnologías en la nube. Los datos con que contamos estiman que en los centros de recursos empresariales hay alojadas 73 empresas emprendedoras. Por lo que no se llevó a cabo, muestreo estadístico, sino que se presentó una encuesta a los responsables de dichas pymes. De las encuestas obtenidas, fueron validadas 71 .

Los encuestados debían poseer orientación emprendedora, que se define como la medida en que una persona es innovadora, proactiva y tomando riesgos (Lee y Peterson, 2000). Se demostró que las personas con una fuerte orientación empresarial son más propensas a adoptar innovaciones tecnológicas, como el cloud computing (Ratten, 2012).

El trabajo de campo se llevó a cabo, entre septiembre y noviembre de 2016 y la obtención de los resultados de los cuestionarios se llevó a cabo con Google Drive, concretamente con la aplicación Google Forms. El cuestionario realizado fue testeado con expertos y cinco empresas que se prestaron a ello. En esa etapa se consiguió detectar algunas ambigüedades y escalas mal planteadas. El $45 \%$ de los cuestionarios fueron obtenidos por medios telefónicos, el $36 \%$ fue presencial y el 19\% mediante correo electrónico. El cuestionario final tuvo un total de 10 preguntas del contenido objetivo de estudio de la investigación y 5 de clasificación. Para valorar aspectos relacionados con la percepción de las ventajas e inconvenientes de los servicios y tecnología cloud se usó la escala Likert 5.

\section{ANÁLISIS DE LOS RESULTADOS}

En el presente apartado, se expondrán los resultados obtenidos a través de la encuesta realizada entre empresas emprendedoras.

\subsection{RIESGOS: CONFIDENCIALIDAD Y FIABILIDAD}

Las empresas encuestadas encuentran que los datos son siempre accesibles (4,2 sobre 5), el acceso a los mismos es rápido (4,5 sobre 5), y que la gestión de los archivos es fácil de usar (4,3 sobre 5$)$.

Por otro lado, las empresas conceden una fiabilidad moderada en cuanto a que los datos serán confidenciales o que no se perderán. En el aspecto de cumplimiento con la legislación en materia de protección de datos, existe cierta desconfianza (medias entre 3 y 4 en todas las empresas sea cual sea su tamaño o antigüedad). 


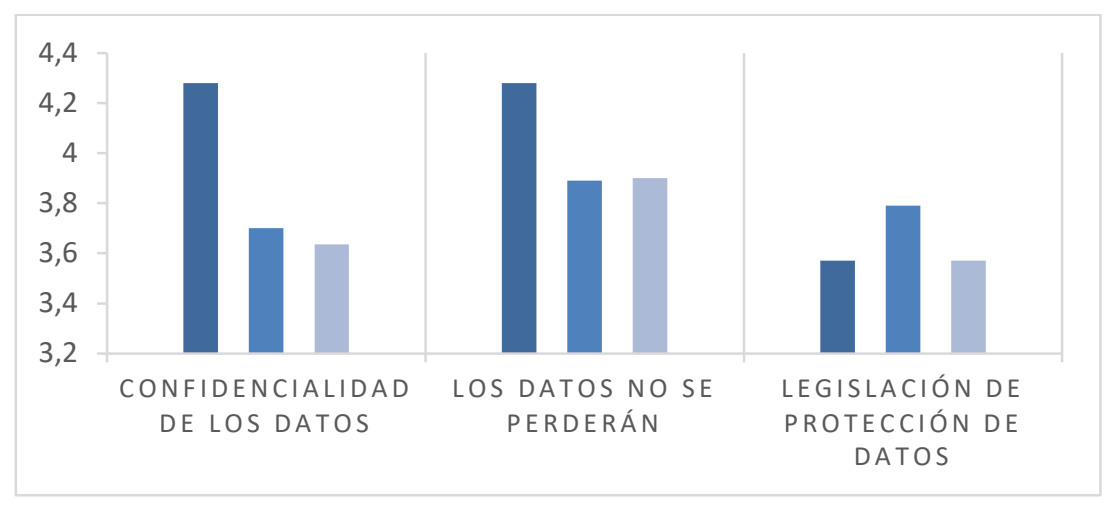

Figura 1. Privacidad, Seguridad y Protección de datos.

Fuente: elaboración propia.

Las empresas con una antigüedad menor a 1 año, muestran más confianza y seguridad en que los datos no se perderán y en la confidencialidad de los mismos (ver figura 1). Mientras que las empresas con una antigüedad superior a 1 año, 3 años depositan menos confianza.

\subsection{VENTAJAS DEL CLOUD}

Las empresas obtienen mayores ventajas en la mejora de la productividad (3,8 sobre 5), seguido de la mayor flexibilidad y escalabilidad de los recursos TIC (3,7 sobre 5$)$ así como la ventaja de disminución de costes en hardware (3,8 sobre 5$)$. Una ventaja destacable es el hecho de que al usar la nube aumenta la capacidad informática sin aumentar los recursos de las empresas ( 3,9 sobre 5$)$.

Otras ventajas que Cloud Computing aporta a las empresas, aunque en menor medida, son: implantación de aplicaciones más rápidas $(3,3)$, ahorro en costes de informática $(3,4)$, el uso de Cloud asegura la continuidad de las operaciones de negocio $(3,3)$ y en menor grado una mejor competencia con empresas que tienen más recursos $(3,2)$.

Por el contrario, las empresas encuentran escasa ventaja en el control de costes y beneficios mediante la utilización de Cloud Computing (2,9 sobre 5).

Analizando algunas de estas ventajas en relación con el tamaño de las empresas, se puede observar que las empresas más pequeñas se benefician en mayor medida de estas ventajas, que las empresas de mayor tamaño

En el $64 \%$ de las empresas encuestadas, el porcentaje de los trabajadores que usan la nube para llevar a cabo sus tareas habituales está comprendido entre el 75 y el $100 \%$. En el $14 \%$ de las empresas, los trabajadores usan la nube para ejecutar sus tareas habituales en un porcentaje comprendido entre el 25 y el $75 \%$. En el $22 \%$ restante de las empresas encuestadas, los trabajadores que usan la nube para ejecutar sus tareas diarias se encuentran en el intervalo del 0 al $25 \%$. 


\subsection{ANÁLISIS DE LA ADOPCIÓN DEL SERVICIO CLOUD POR PARTE DE LAS EMPRESAS}

El $41 \%$ de las empresas encuestadas comenzaron a usar el servicio Cloud Computing gracias a recomendaciones profesionales. El 19\% de las empresas lo hicieron por recomendaciones o sugerencias de amigos o conocidos. Un porcentaje importante de las empresas encuestadas (24\%) comenzaron a usar la nube por otros medios.

Otros factores de influencia que hicieron que adoptaran el Cloud, fueron: los medios de comunicación (6\%), publicidad comercial (4\%), y un mínimo porcentaje de empresas $(2 \%)$ lo hicieron gracias a cursos de formación, ofertas comerciales y redes sociales.

En cuanto a la utilidad que dan las empresas al servicio de la nube, la que más usan las empresas son los ficheros y bases de datos, ya que según los resultados de la encuesta, son usados por el $71 \%$ de las empresas. La nube es usada para tareas de ofimática en el $58 \%$ de las empresas. También un importante porcentaje de uso le corresponde a tareas de ERP (contabilidad y facturación) y para la gestión de clientes (CRM). El $40 \%$ de las empresas encuestadas usan la nube para estas tareas.

El servicio de la nube también es útil para las empresas en aspectos de desarrollo ( $40 \%$ de empresas lo usan) Hosting (36\%), E-Commerce (31\%) y ventas (22\%). Las utilidades de Proyectos Nóminas, RRHH y Logística tienen un uso escaso según las empresas encuestadas, ya que sólo un $9 \%$, un $13 \%$ y un $16 \%$ respectivamente, las usan para su actividad ordinaria.

El servicio de Google Drive es usado en aproximadamente casi el $80 \%$ de las empresas encuestadas. Sin duda, el servicio que más se usa en las empresas es Dropbox, ya que más del $80 \%$ de las empresas encuestadas hacen uso de este servicio. Por el contrario, los servicios menos utilizados por las empresas son Asus y Webstorage que escasamente llegan al $20 \%$ de uso.

El servicio iCloud se encuentra en una posición baja pasando del $30 \%$ de uso pero sin llegar al $40 \%$. Por otra parte, otros servicios de la nube usados por las empresas alcanzan el $40 \%$ de uso entre las empresas encuestadas.

\subsection{VALORACIÓN MEDIA DE LOS SERVICIOS CLOUD}

En cuanto al grado de valoración de los servicios expuestos, cabe señalar que el más valorado es Dropbox con 4,7 puntos sobre 5, le sigue Google Drive con una puntuación de 3,9 sobre 5. Otro de los servicios que destaca por su valoración es iCloud (3,7 puntos sobre 5).

Así como otros servicios, no expuestos explícitamente, que las empresas valoran de manera significativa, 4,1 sobre 5 . Por el contrario, los servicios menos valorados son Asus y Webstorage con 2,2 y 2,7 puntos sobre 5 respectivamente. 
En cuanto a los tipos de ficheros que depositan en la nube, de manera habitual se trata de documentos generales, con una frecuencia media de 4,2 sobre 5 , con menor frecuencia se depositan documentos importantes (3,4 sobre 5$)$.

Las fotos, correo o e-mail también son archivos que se depositan con regularidad en las empresas encuestadas, con una frecuencia de 3,3 3,1 y 3,2 respectivamente sobre 5 . Se depositan con menor regularidad archivos de software o audio y videos, con una frecuencia de 2,1 y 2,4 sobre 5 respectivamente.

\section{CONCLUSIONES}

Según los datos obtenidos las principales ventajas que perciben las empresas con el uso de Cloud Computing, recae en la mejora de la productividad, la mejor flexibilidad y escalabilidad de los recursos TIC y la disminución de costes en hardware. Estos datos coinciden con estudios anteriores (Armbrust et al., 2010).

Según los resultados obtenidos, las empresas con un menor número de trabajadores se benefician en mayor medida de estas ventajas que las empresas con más trabajadores. Este hecho también se confirma en las empresas que tienen menos antigüedad y que por tanto están en una fase más emprendedora, lo que nos lleva a confirmar que existe relación entre grado de emprendimiento, capacidad emprendedora y adopción Cloud (Ratten, 2013).

Ahora bien, es la confianza y la seguridad en el servicio de la nube para la realización de su actividad ordinaria. En los resultados obtenidos se observa que las empresas otorgan cierto grado de confianza en los servicios de la nube, creen en la confidencialidad de los datos y en que estos no se perderán, aunque no depositan una total confianza. Las pymes analizadas se encuentran más desconfiadas en que se cumpla la legislación en materia de protección de datos. Por otro lado, sí están de acuerdo en la rápida accesibilidad de los datos, así como su fácil gestión gracias al servicio de la nube. Se confirma que existe relación entre grado de emprendimiento y uso del Cloud, ya que son las empresas más jóvenes las que mayor confianza depositan, mientras que las que superan los 3 años de existencia se muestran más reacias a confiar en esta tecnología.

De los servicios expuestos en el cuestionario los más utilizados por las empresas encuestadas son Dropbox y Google Drive. Las pymes valoran en mayor medida las aplicaciones de Dropbox y Google Drive, seguido del servicio iCloud aunque también otorgan gran valoración a otros servicios que no aparecen como opción en el cuestionario, como pueden ser: Box, Hubic, Tresorit o Mega.

En cuanto al tipo de archivo que se deposita en el almacenamiento de la nube, es muy habitual el almacenamiento de documentos generales, en menor medida se hace con documentos importantes. 
La utilidad que dan las empresas al servicio de la nube, se concentra mayoritariamente en tareas de ofimática y ficheros y bases de datos. También se usa con gran frecuencia, aplicaciones de ERP, así como que permiten recopilar datos de clientes, como el perfil, hábitos de consumo u otros datos de interés para la gestión de la organización.

En cuanto al porcentaje de trabajadores que usan la nube, cabe decir que la mayoría de empresas que usan la nube, lo hace de manera completa, es decir que todos o gran parte de sus trabajadores usan los servicios de la nube para sus tareas habituales. También decir que, en las empresas con menor número de trabajadores, estos usan la nube en un porcentaje del 75 al 100\%, en cambio en las empresas con mayor número de trabajadores este porcentaje se reduce hasta situarse entre un 25 y $50 \%$. Con lo que se confirma que el tamaño de la organización, medido en términos de no de trabajadores influye en la intención de adoptar la nube, coincidiendo con otras investigaciones (Palos y Correia, 2016). Por tanto, estas conclusiones apoyan la premisa de que las tecnologías Cloud facilitan el emprendimiento, y la innovación, lo que está en línea con investigaciones anteriores (Ross y Blumenstein, 2015).

Este soporte al emprendimiento que podría suponer la tecnología Cloud, supondría para las empresas emprendedoras contar con la posibilidad de utilizar los servicios de Cloud para desarrollar y vender sus propios productos. Por tanto, las pymes que desde su creación han utilizado estrategias basadas en la nube "nativas de la nube", podrían desarrollar su actividad productiva en mejores condiciones que las empresas existentes, las cuales se enfrentan al reto de la adopción Cloud. 


\section{REFERENCIAS BIBLIOGRÁFICAS}

Alshamaila, Y., Papagiannidis, S., y Li, F. (2013). Cloud computing adoption by SMEs in the north east of England: A multi-perspective framework. Journal of Enterprise Information Management, 26(3), 250-275.

Armbrust, M., Fox, A., Griffith, R., Joseph, A. D., Katz, R., Konwinski, A., ... y Zaharia, M. (2010). A view of cloud computing. Communications of the ACM, 53(4), 50-58.

Arinze, B., y Anandarajan, M. (2012). Factors that determine the adoption of cloud computing: A global perspective. Enterprise Information Systems and Advancing Business Solutions: Emerging Models: Emerging Models, 21

Benlian, A., y Hess, T. (2010). The Risks of Sourcing Software as a Service-An Empirical Analysis of Adopters and Non-Adopters. In ECIS.

Benlian, A., y Hess, T. (2011). Opportunities and risks of software-as-a-service: Findings from a survey of IT executives. Decision Support Systems, 52(1), 232-246.

Brodkin, J. (2008). Gartner: Seven cloud-computing security risks. Infoworld,2008, 1-3.

Fundación Innovación Bankinter (2010). Cloud Computing-La tercera ola de las Tecnologías de la Información (Fundación Innovación Bankinter-Accenture).

Guilarte, M. (2015). Muy Cloud. Recuperado el 7 de Marzo de 2016, de http://muycloud.com/2013/07/19/aplicaciones-cloud-pymes/

Gupta, P., Seetharaman, A., y Raj, J. R. (2013). The usage and adoption of cloud computing by small and medium businesses. International Journal of Information Management, 33(5), 861-874.

Heiser, J., y Nicolett, M. Assessing the Security Risks of Cloud Computing, Gartner Report. 2008.

Hooper, C., Martini, B., y Choo, K. K. R. (2013). Cloud computing and its implications for cybercrime investigations in Australia. Computer Law \& Security Review, 29(2), 152163.

IDC. (2011). Cuando las empresas se rinden al Cloud. International Data Corporation.

Lee, S.M. y Peterson, S.J., 2000. Culture, entrepreneurial orientation, and global competitiveness. Journal of World Business 35 (4), 401-416.

Leimeister, S., Böhm, M., Riedl, C., y Krcmar, H. (2010, June). The Business Perspective of Cloud Computing: Actors, Roles and Value Networks. In ECIS. 
Lian, J. W., Yen, D. C., y Wang, Y. T. (2014). An exploratory study to understand the critical factors affecting the decision to adopt cloud computing in Taiwan hospital. International Journal of Information Management, 34(1), 28-36.

Low, C., Chen, Y., y Wu, M. (2011). Understanding the determinants of cloud computing adoption. Industrial management \& data systems, 111(7), 1006-1023.

Maqueira-Marín, J. M., y Bruque-Cámara, S. (2012). Drivers of Cloud Computing Adoption in Companies: Who moves the Cloud?. Universia Business Review, (35), 56.

Marston, S., Li, Z., Bandyopadhyay, S., Zhang, J. y Ghalsasi, A., (2011). Cloud computing - the business perspective. Decis. Support. Syst. 51 (1), 176-189.

Martín. E (2 de diciembre de 2014).TIC beat.Recuperado el 16 de marzo de 2016.de http://www.ticbeat.com/cloud/que-es-cloud-computing-definicion-concepto-paraneofitos/

Mell, P. y Grance, T. (2011). The NIST definition of cloud computing

NIST. (2011). National Institute of Standards and Technology.

Oppenheim, C. (2012). Cloud law and contract negotiation. El profesional de la información, 21(5), 453-457

Palos, P. R., y Aguayo-Camacho, M. (2016). Los cambios organizacionales y la nube en el entorno turístico: estudio de caso. International Journal of World of Tourism, Vol. 3, № 5, pp 33-42.

Palos, P.R. y Correia, M.B. (2016). La actitud de los recursos humanos de las organizaciones ante la complejidad de las aplicaciones SaaS. Dos Algarves: A Multidisciplinary eJournal, 28, 87-103. doi: 10.18089/DAMeJ.2016.28.6

Palos-Sanchez, P.R. (2017). CLOUD COMPUTING: seguridad, privacidad y reputación online del sector empresarial turístico. Revista TURyDES, Turismo y Desarrollo Local, Vol. 10 (22).

Patel, P., Ranabahu, A. H., y Sheth, A. P. (2009). Service level agreement in cloud computing.

Ratten, V. (2012). Entrepreneurial and ethical adoption behaviour of cloud computing. The Journal of High Technology Management Research, 23(2), 155-164.

Ratten, V. (2013). Cloud computing: A social cognitive perspective of ethics, entrepreneurship, technology marketing, computer self-efficacy and outcome expectancy on behavioural intentions. Australasian Marketing Journal (AMJ), 21(3), 137-146.

Reyna, J. E. V. (2009). Cloud Computing. La computadora, herramienta indispensable en diversas áreas de conocimiento, 161. 
Ross, P. K., y Blumenstein, M. (2012). Leveraging the opportunities of the Cloud: The impact of Cloud Computing strategies on Organisational Structures, Management Practices and ICT Worker Skill Sets in Queensland private and public sector organisations. Griffith University and the Department of Science, Information, Technology, Innovation and The Arts (SITIA).

Ross, P., y Blumenstein, M. (2013). Cloud computing: the nexus of strategy and technology. Journal of Business Strategy, 34(4), 39-47.

Ross, P.K. y Blumenstein, M. (2015). Cloud computing as a facilitator of SME entrepreneurship, Technology Analysis \& Strategic Management, 27:1, 87-101, DOI: 10.1080/09537325.2014.951621

Ryan, W. M., \& Loeffler, C. M. (2010). Insights into cloud computing. Intellectual Property \& Technology Law Journal, 22(11), 22.

Tuncay, E. (2010). Effective use of cloud computing in educational institutions. Procedia Social and Behavioral Sciences, 2, 938-42.

Urueña, A., Ferrari, A., Blanco, D., y Valdecasa, E. (2012). Cloud Computing Retos y Oportunidades. ONTSI, Mo de Industria de España, Pág, 18.

Valencia, K. (2012). Historia del Cloud Computing. Revista de Informacion Tecnológica y Sociedad, pp.51-52.

Wu, D., Schaefer, D., y Rosen, D. W. (2013). Cloud-based design and manufacturing systems: a social network analysis. In International Conference on Engineering Design, ICED 2013. University of Bath.

Yigitbasioglu, O., Mackenzie, K., y Low, R. (2013). Cloud Computing: How does it differ from IT outsourcing and what are the implications for practice and research?. The International Journal of Digital Accounting Research, 13, 99-121.

Yigitbasioglu, O. M. (2015). The role of institutional pressures and top management support in the intention to adopt cloud computing solutions. Journal of Enterprise Information Management, 28(4), 579-594. 